\title{
Temporal and spatial distribution of the aquaporin 1 in spinal cord and dorsal root ganglia after traumatic injuries of the sciatic nerve
}

\author{
Yasemin Kaya • Umut Ozsoy • Necdet Demir • \\ Arzu Hizay • L. Bikem Suzen • Doychin N. Angelov • \\ Levent Sarikcioglu
}

Received: 7 April 2014 / Accepted: 12 June 2014 / Published online: 15 August 2014

(C) Springer-Verlag Berlin Heidelberg 2014

\begin{abstract}
Purpose The aquaporin family comprises a large family of integral membrane proteins that enable the movement of water and other small, neutral solutes across plasma membranes. Although function and mechanism of aquaporins in central nervous system injury have been reported, the pathophysiologic role of aquaporin 1 (AQP1) in peripheral nerve has not been extensively documented. In the present study, we aimed to study the temporal and spatial distribution of AQP1 in spinal cord and dorsal root ganglia after sciatic nerve injury. Methods Forty-eight adult female mice were randomly divided into four groups (intact controls, sham operated, cut injury, and crush injury). Animals receiving cut or crush injuries were sacrificed at the 2nd, 24th, and 48th postoperative hours. Spinal cord samples at the level of lumbosacral intumescences and corresponding dorsal root ganglia on the experimental and contralateral side were dissected free and proceeded to AQP1 immunohistochemistry.

Results Our quantitative estimations revealed that a sharp increase in AQP1 immunoreactivity at the 24th postoperative hour was observed. This sharp increase was no more evident at $48 \mathrm{~h}$ after sciatic nerve injury. Identical peak was observed after both cut and crush injuries.
\end{abstract}

Y. Kaya $\cdot$ U. Ozsoy • A. Hizay $\cdot$ L. B. Suzen • L. Sarikcioglu ( $\bowtie)$ Department of Anatomy, Akdeniz University Faculty of Medicine, Antalya 07070, Turkey

e-mail: levent@akdeniz.edu.tr

L. Sarikcioglu

e-mail: sarikcioglul@gmail.com

N. Demir

Department of Histology and Embryology, Akdeniz University

Faculty of Medicine, Antalya 07070, Turkey

D. N. Angelov

Anatomical Institute, University of Cologne, Cologne, Germany
Conclusions We demonstrated that there was a temporal relationship with an increased expression of AQP1 following injury sustained to the sciatic nerve that was significantly observed in dorsal root ganglia and spinal cord. Those expressions were also subsided over time.

Keywords Aquaporin 1 - Spinal cord · Dorsal root ganglia * Sciatic nerve injury

\section{Introduction}

The aquaporins family comprise a large family of integral membrane proteins that enable the movement of water and other small, neutral solutes across plasma membranes [1]. The function of these proteins is to control particular aspects of homeostasis [2,3]. The aquaporins (AQP) family in the central nervous system has diverse functions in neural signal transduction, cerebrospinal fluid formation, and osmoreception [4]. It has been reported that maintenance of osmotic composition and volume within the interstitial, glial, and neuronal compartments of the central nervous system are essential for normal function. Even small changes in osmolarity or volume can dramatically alter neuronal signaling and information processing $[5,6]$. It has been documented in the current literature that $\mathrm{AQP} 1, \mathrm{AQP} 4$, and $\mathrm{AQP} 9$ play important roles in brain water homeostasis [7-10].

Gao et al. [11] showed that AQP1 is main water channel in the peripheral nervous system. In the peripheral nervous system, AQP1 has been found to be expressed in trigeminal ganglion cells, periodontal cells, Ruffini endings, glial cells, dorsal root ganglia, terminal Schwann cells, nodose ganglion cells, and the enteric nervous system [10,12-14]. Although function and mechanism of aquaporins in central nervous system 
injury have been reported, the pathophysiologic role of AQP1 in peripheral nerve has not been extensively documented. Therefore, further investigations in wellknown traumatic injuries of the peripheral nervous system are required to understand the temporal and spatial distribution of AQP1 in spinal cord and dorsal root ganglia and possible role (s) of AQP1 in intricate processes of the peripheral nerve injury/repair.

\section{Material and method}

Number, strain, and sex of animals

Forty-eight adult female BalbC mice were obtained from the Laboratory Animal Unit of the Akdeniz University. Animals were housed in cages under standard environmental conditions (light between 06:00 and 18:00 h, temperature at $22{ }^{\circ} \mathrm{C}$, and free access to chow and water). All experimental protocols were approved by the Animal Welfare Committee of the Akdeniz University (protocol number 2013.05.04) and conducted in accordance with Turkish Law on the Protection of Animals.

\section{Animal groups and experimental design}

Study groups are depicted in Table 1. Throughout the study, allocation was concealed, i.e., the person undertaking the surgery did not know to which group the animal would be allocated. Animals were randomized into groups using a randomized number sequence and assessment was blinded.

1. Group 1 (intact control, $\mathrm{n}=6$ ). In these animals, no surgical procedures were performed. They were intact mice.

2. Group 2 (sham-operated, $n=6$ ). Following sufficient anesthesia, skin of the lateral surface of the left thigh was incised in this group. Left sciatic nerve was exposed by a hind limb muscle splitting approach. After viewing the sciatic nerve, skin was immediately sutured with a 5.0 Ethilon ${ }^{\circledR}$ suture and mice were allowed to recover in a postoperative room.

3. Group 3 (cut injury, $n=18$ ). In these animals, cut injury was performed. The animals were then randomized into groups using a randomized number sequence. Groups $3 \mathrm{a}$, $3 \mathrm{~b}$, and $3 \mathrm{c}$ were sacrificed at the end of the $2 \mathrm{nd}, 24 \mathrm{th}$, and 48th postoperative hours, respectively (Table 1 ).

4. Group 4 (crush injury, $n=18$ ). In these animals, cut injury was performed. The animals were then randomized into groups using a randomized number sequence. Groups $4 \mathrm{a}$, $4 \mathrm{~b}$, and $4 \mathrm{c}$ were sacrificed at the end of the $2 \mathrm{nd}, 24 \mathrm{th}$, and 48th postoperative hours, respectively (Table 1 ).
Table 1 Study groups

\begin{tabular}{|c|c|c|}
\hline- & $\mathrm{n}$ & Subgroups \\
\hline Group 1 (control) & 6 & Intact mice \\
\hline Group 2 (sham operated) & 6 & $\begin{array}{l}\text { Skin opened, no nerve cut } \\
\text { and suture }\end{array}$ \\
\hline \multirow[t]{3}{*}{ Group 3 (cut injury) } & 18 & $\begin{array}{l}\text { Group 3a: second postoperative } \\
\text { hour ( } 6 \text { animals) }\end{array}$ \\
\hline & - & $\begin{array}{l}\text { Group 3b: } 24 \text { th postoperative } \\
\text { hour ( } 6 \text { animals) }\end{array}$ \\
\hline & - & $\begin{array}{l}\text { Group 3c: } 48 \text { th postoperative } \\
\text { hour ( } 6 \text { animals })\end{array}$ \\
\hline \multirow[t]{3}{*}{ Group 4 (crush injury) } & 18 & $\begin{array}{l}\text { Group 4a: second postoperative } \\
\text { hour ( } 6 \text { animals })\end{array}$ \\
\hline & - & $\begin{array}{l}\text { Group 4b: } 24 \text { th postoperative } \\
\text { hour ( } 6 \text { animals) }\end{array}$ \\
\hline & - & $\begin{array}{l}\text { Group 4c: 48th postoperative } \\
\text { hour ( } 6 \text { animals) }\end{array}$ \\
\hline
\end{tabular}

Surgical procedures

After being prepared for aseptic surgery, animals were anesthetized with a mixture of xylazin HCI $(15 \mathrm{mg} / \mathrm{kg}$, Alfazyne, Alfasan International B.V., Woerden, Holland) and ketamine (100 mg/kg, Ketasol, Richter Pharma AG, Wels, Austria) via intramuscular injection. Following sufficient anesthesia, skin of the lateral surface of the left thigh was incised. A hind limb muscle splitting approach was used to expose the left sciatic nerve and its three branches under magnification with a fiberoptic-illuminated operating microscope (Olympus SZ61). Careful blunt dissection over a length of 1 to $1.5 \mathrm{~cm}$ was performed to isolate the sciatic nerve from the surrounding connective tissue. The nerve was cut transversely at the level of midthigh and repaired immediately by three to four stitches with 10.0 suture material (Fig. 1). Following the nerve cut and suture, muscle and skin layers were immediately sutured with a 5.0 Ethilon ${ }^{\circledR}$ suture and mice were allowed to recover in a postoperative room.

\section{Tissue preparation}

At the end of postoperative period (Table 1), animals were perfused (0.1 M phosphate buffered saline, $\mathrm{pH} 7.4$; PBS) and fixed (4\% paraformaldehyde in $0.1 \mathrm{M}$ phosphate-buffered saline, $\mathrm{pH}$ 7.4) transcardially. Following perfusion-fixation procedure, spinal cord samples at the level of lumbosacral intumescences and corresponding dorsal root ganglia on the experimental and contralateral side were dissected free and kept in $4 \%$ paraformaldehyde in $0.1 \mathrm{M}$ phosphate buffered saline, $\mathrm{pH} 7.4$ (in dark and $+4{ }^{\circ} \mathrm{C}$ ).

All samples were postfixed in $4 \%$ paraformaldehyde overnight and cryoprotected in $20 \%$ sucrose in PBS. Transverse sections $(30 \mu \mathrm{m}$ thick for spinal cord, 5-7 $\mu \mathrm{m}$ thick for dorsal 


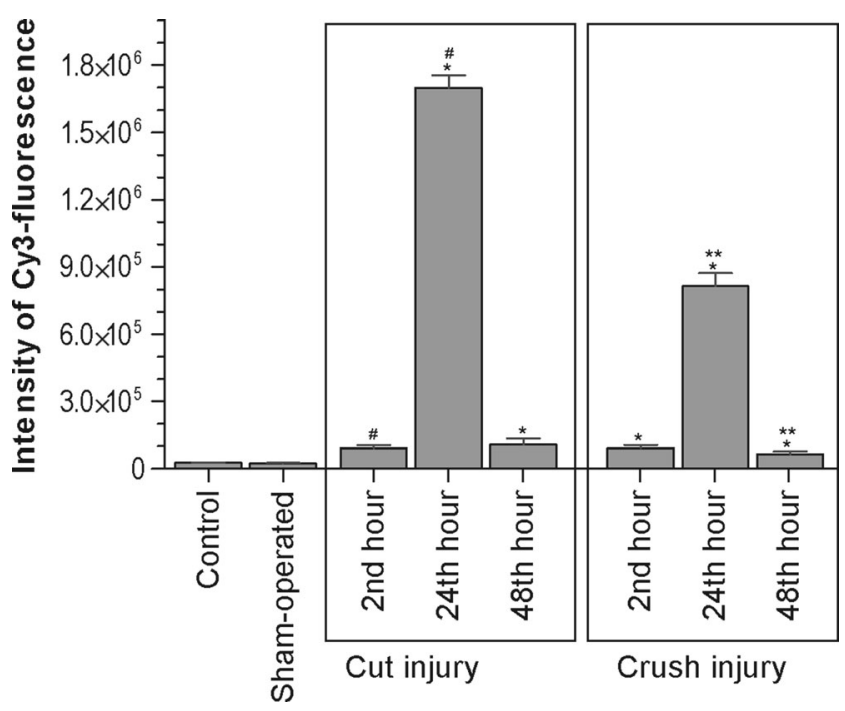

Fig. 1 AQP1 immunofluorescence intensity in dorsal root ganglia samples after cut and crush injuries of the sciatic nerve. ${ }^{*} p<0.05$, significant difference versus intact group; $\# p<0.05$, significant difference versus second postoperative hour after cut injury (group $3 \mathrm{a}$ ); $* * p<0.05$, significant difference versus second postoperative hour after crush injury (group 4a)

root ganglia) were cut on a cryostat and mounted on SuperFrost Plus slides (Art. No. J1800AMNZ, Menzel-Gläser, Germany). The tissue slices were stored at $-80{ }^{\circ} \mathrm{C}$ until immunostaining. Using the fractionator principle, every third section of a total of 30-39 sections through the spinal cord and every second section out of 9-12 dorsal root ganglia sections was processed for immunohistochemistry.

\section{Immunofluorescence}

Sections were air-dried and rinsed $(3 \times 10 \mathrm{~min}$ in $0.1 \mathrm{M}$ PBS; pH 7.4). Nonspecific binding was blocked using PBS containing $0.2 \%$ Triton X-100 (Sigma, St. Louis, MO, USA), $0.02 \%$ sodium azide (Merck, Darmstadt, Germany), and $5 \%$ normal sheep serum (NSS) for $30 \mathrm{~min}$ at room temperature. Sections were incubated with an unconjugated polyclonal antibody to AQP1 (sc-20810, Santa Cruz Biotechnology) overnight at $4{ }^{\circ} \mathrm{C}$. Sections were then incubated with a goat polyclonal secondary antibody to rabbit IgG-H\&L (Texas Red) (ab6719, Abcam, Cambridge, UK). After immunofluorescence labeling, sections were mounted and coverslipped with mounting medium (Vectashield H-1200, Vector Laboratories, Burlingame, CA, USA). Controls were performed by omitting primary antibody. Control sections showed no detectable labeling. Slides were observed with a Zeiss Axioskop 50 epifluorescence microscope through "rhodamine" (no. 15 of Carl Zeiss) and "fluorescein" (no. 9 of Carl Zeiss) filters.
Digital image analysis for intensity of fluorescence

Fluorescent images were compared using the 8-BPP grayscale format. Thereby, each pixel contains eight bits of information encoding brightness, which ranges in a scale from 0 to 255 . The scale for pixel brightness, or pixel gray value, is constructed so that the higher numbers indicate greater pixel brightness. Digital images were captured with a slow scan CCD camera (DFC345FX, Leica Microsystems, Oberkochen, Germany). For quantification of pixel brightness, images were captured using the $\times 40$ objective and the Image-Pro Plus Software Version 6.2 (Media Cybernetics, Inc., Silver Spring, MD, USA). Exposure time was optimized to ensure that only few pixels were saturated at 255 Gy values. However, all images representing the same labeling were taken under same conditions (Exposure, Gain, Gamma).

An identical and constant area of interest (AOI) was selected in both spinal cord and dorsal root ganglia sections. Interactive threshold was used to detect the pixel brightness of the minimum fluorescence. Threshold values ensured the inclusion of the entire signal range in the sample. This value was further used to extract and compare the pixel number between animals of the same group as between experimental groups.

Since at a gray value over 221 just a few pixels representing labeling were present, only pixel grayscale ranges with the maximum change in fluorescence were considered. This threshold was set to a pixel gray value of 220. Accordingly, the range for measuring pixel number and brightness was set at 121-220.

\section{Statistics}

Data on intensity of fluorescence of the AQP1 were presented as mean \pm standard deviation and analyzed by Kruskal-Wallis test (post hoc Dunnet test). The level of significance was set to $\mathrm{p}<0.05$. For analysis, GraphPad Prism version 5.0 (GraphPad Software, Inc, San Diego, CA, USA) was used.

\section{Results}

\section{Dorsal root ganglia}

In intact and sham-operated mice, the intensity of fluorescence measured 27,091 $\pm 2,909$ and 25,704 $\pm 4,211$ (mean \pm $\mathrm{SD} ; \mathrm{N}=6$ ), respectively. Performance of cut injury resulted in a 3.5-fold increase in the AQP1 immunofluorescence intensity at the second postoperative hour $(91,890$ $\pm 30,403)$ as compared to those of intact animals $(27,091 \pm$ 2,909, mean $\pm \mathrm{SD} ; \mathrm{N}=6$ ).

At the 24th postoperative hour, this increase reached 63fold $(1,699,441 \pm 96,736$, mean $\pm S D ; N=6)$. At the 48th 
postoperative hour, a substantial decrease $(\sim 16$-fold $)$ was observed as compared to peak level observed at the 24th postoperative hour. In spite of this substantial decrease, AQP1 immunofluorescence intensity was not regressed to the intact level (Figs. 1 and 2).

Crush injury induced a slow increase in AQP1 immunofluorescence intensity in parallel to cut injury groups. At the second postoperative hour, fluorescence intensity was increased to 3.4-fold $(92,339 \pm 26,365$, mean $\pm \mathrm{SD} ; \mathrm{N}=6)$ compared to those of control group. At 24th postoperative hour, AQP1 immunofluorescence showed a peak $(815,012 \pm$ 115,536 , mean $\pm \mathrm{SD} ; \mathrm{N}=6$ ) 30 -fold increment compared to the intact group).

At the 48th postoperative hour $(66,422 \pm 22,926$, mean \pm $\mathrm{SD} ; \mathrm{N}=6$ ), a substantial decrease ( $\sim 9$-fold) was observed as compared to peak level observed at the 24th postoperative hour. However, it was significantly higher than the intact group (Figs. 1 and 2).

Contralateral dorsal root ganglia were also harvested and analyzed. Intact and sciatic nerve-injured groups (groups 3 and 4) showed approximately similar intensity of fluorescence in comparison to those of the shamoperated group. AQP1 immunofluorescence intensity in the experimental side $(25,704 \pm 4,211)$ and contralateral side $(26,503 \pm 3,288)$ of the sham-operated group showed no significant difference $(\mathrm{p}>0.05)$.

Dorsal horn of the spinal cord

In intact and in sham-operated mice, the intensity of fluorescence in the dorsal horn was measured as measured 27,091 \pm $2,909$ and $25,704 \pm 4,211$ (mean $\pm \mathrm{SD} ; \mathrm{N}=6)$, respectively.

The AQP1 immunofluorescence intensity showed a trend to increase after induction of the both types of sciatic nerve injury. After a moderate increment at the second postoperative hour $(4,315 \pm 535$ in cut injury and 5,421 $\pm 1,436$ in crush injury, mean $\pm \mathrm{SD} ; \mathrm{N}=6$ ), the highest peak was found at the 24th postoperative hour in both injury types $(37,704 \pm 1,525$ in cut injury, 19,289 $\pm 2,606$ in crush injury). Peak trend was not observed at the 48th postoperative hour $(25,061 \pm 2,789$ in cut injury and 14,181 $\pm 1,094$ in crush injury, mean $\pm \mathrm{SD} ; \mathrm{N}=6$ ) but it was still higher than those of the second postoperative hour (Fig. 3).

In animals of groups 3 and 4, AQP1 immunofluorescence intensity in the superficial laminae I and II of the dorsal horn (Fig. 4), as well as in attached dorsal root ganglia (Fig. 2) was stronger than those of intact animals. Alterations in AQP1 immunofluorescence intensity seen in dorsal root ganglia

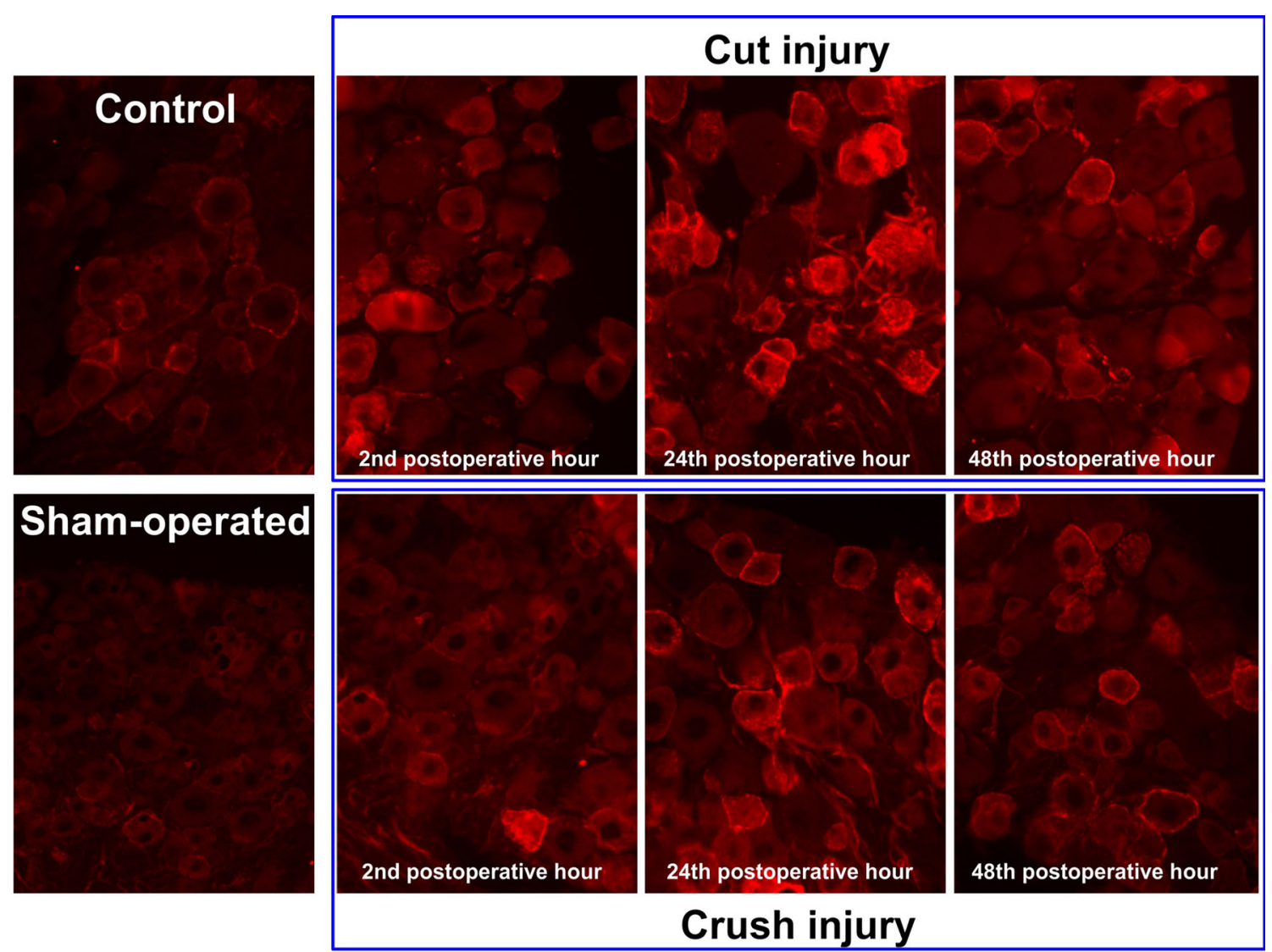

Fig. 2 Micrographs showing appearance of AQP1 immunofluorescence intensity in dorsal root ganglia samples after cut and crush injuries of the sciatic nerve 
Fig. $3 \mathrm{AQP} 1$ spinal cord samples after cut and crush injuries of the sciatic nerve immunofluorescence intensity in

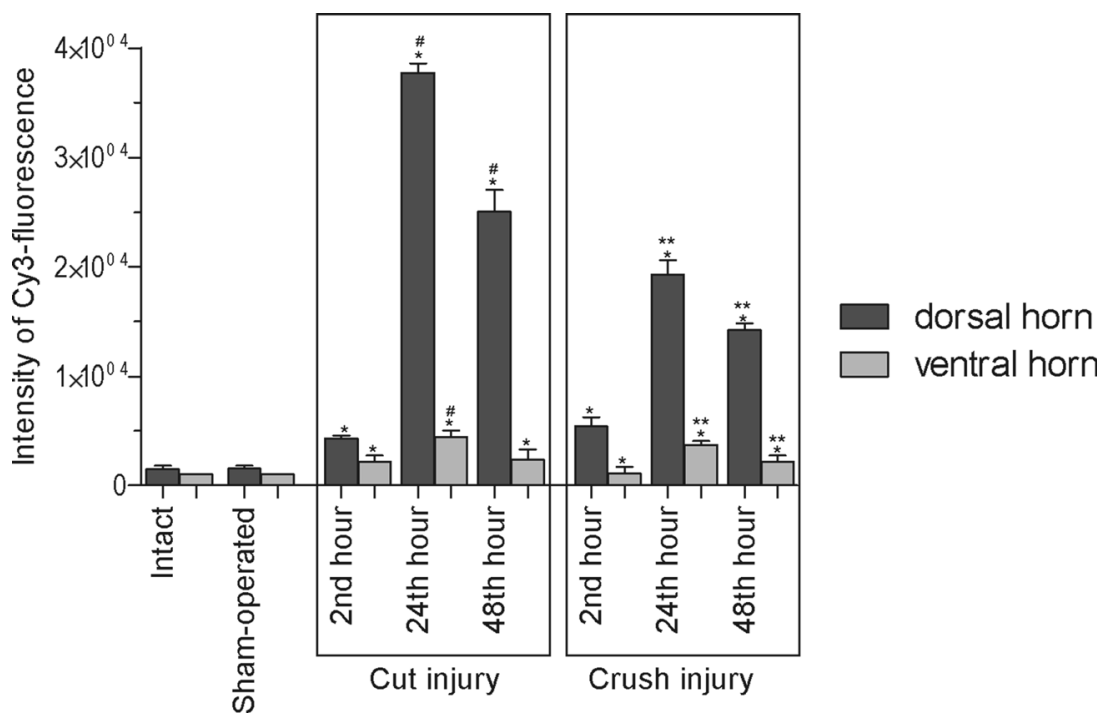

were also observed in ventral and dorsal horns of the spinal cord samples.

\section{Ventral horn of the spinal cord}

In intact and in sham-operated mice, the intensity of fluorescence in the ventral horn was measured as measured 27,091 \pm 2,909 and 25,704 $\pm 4,211$ (mean $\pm \mathrm{SD} ; \mathrm{N}=6$ ), respectively. Intensity of fluorescence of AQP1 immunoreactivity in the ventral horn samples were in similar direction but lower in magnitude than those of dorsal horn (Fig. 3). The AQP1 immunofluorescence intensity also showed a trend of increase after induction of the both types of sciatic nerve injury. After a moderate increment at the second postoperative hour $[2,193 \pm$ 977 (mean $\pm \mathrm{SD} ; \mathrm{N}=6$ ) in cut injury and 1,103 \pm 191 (mean \pm $\mathrm{SD} ; \mathrm{N}=6$ ) in crush injury], the highest peak was found at the 24th postoperative hour in both injury types [4,455 $\pm 1,172$ (mean $\pm \mathrm{SD} ; \mathrm{N}=6$ ) in cut injury and 3,705 \pm 776 (mean $\pm \mathrm{SD}$; $\mathrm{N}=6$ ) in crush injury]. Peak trend was not observed at the 48th postoperative hour $[2,381 \pm 1,585$ (mean $\pm \mathrm{SD} ; \mathrm{N}=6)$ in cut injury and 2,193 \pm 977 in crush injury] but it was still higher than those of the second postoperative hour (Fig. 3).

\section{Discussion}

In the present study, we focused on the dorsal root ganglia and ventral-dorsal horn of the spinal cord at the level of lumbosacral intumescence and studied the temporal and spatial distribution of the AQP1 after sciatic nerve injuries. We found that AQP1 immunoreactivity had a sharp increase at the 24th postoperative hour. This sharp increase did not continue at the 48th hour after induction of the sciatic nerve injury.

\section{Biological role of $\mathrm{AQP1}$}

Water is the single most abundant substance in cells and organisms and is an important molecule involved in several biochemical processes present in living cells [15]. Molecular
Fig. 4 Micrographs showing appearance of AQP1 immunofluorescence intensity in spinal cord samples 24th postoperative hour after cut injury of the sciatic nerve. a Larger view of the superficial laminae of the dorsal horn, $b$ low power micrograph with schematic drawings on spinal cord transeverse section. Gray matter delineated (white lines), $\mathrm{A}$ and $\mathrm{C}$ (blue frames) magnified in a and c, respectively. c Larger view of the ventral horn
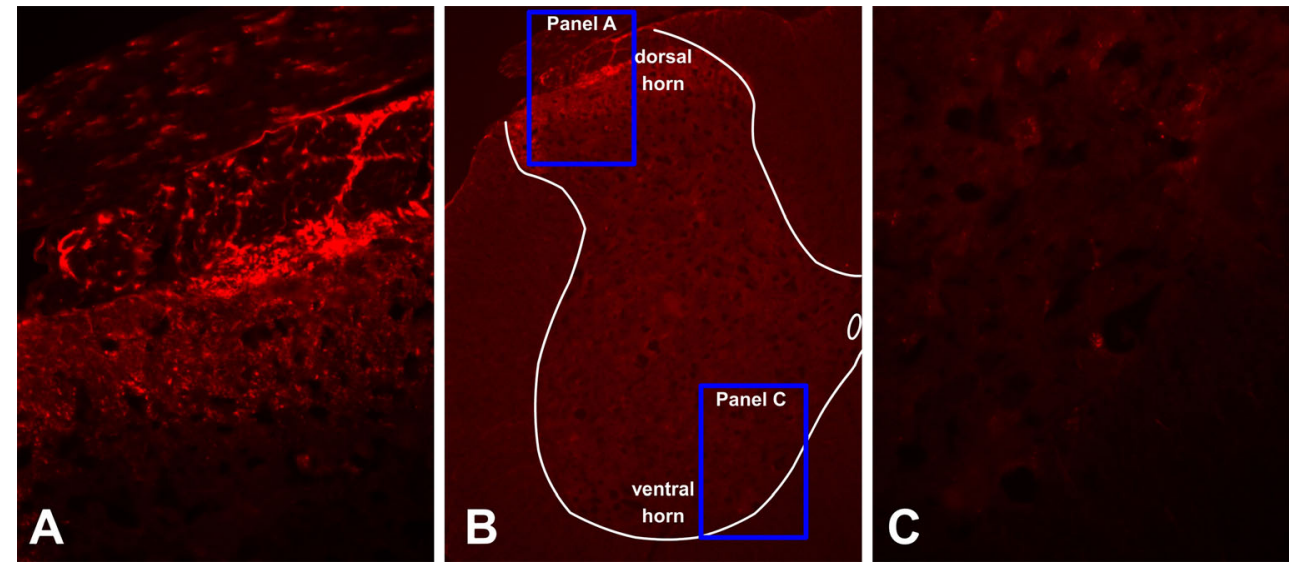
identification of the AQP1 in red blood cells and renal tubules has characterized a new stream of research. Classical roles of the aquaporins in facilitating trans-epithelial fluid transport is well understood, as in the urinary concentrating mechanism and gland fluid secretion, while the molecular mechanisms to regulate water permeability in the nervous system are still unclear [15]. Analysis of AQP-deficient mice revealed some evidences on the involvement of aquaporins in a variety of physiological and cellular functions. Roles of AQPs in transepithelial fluid transport and tissue swelling has also been studied, and a diverse set of unanticipated cellular roles of aquaporins has been reported. Their involvement in cell migration, fat metabolism, epidermal biology, and neural signal transduction may be important in the pathophysiology of cancer, obesity, immune cell dysfunction, and epilepsy $[2,3]$.

Neurobiological relevance of AQP1 increase after peripheral nerve injury

Although expression and localization of aquaporins in the central nervous system have been documented, there are few studies on their existence in the peripheral nervous system [15]. AQP1 has been reported to be expressed by amacrine cells of the rat retina [16], Dogiel type I neurons of the enteric nervous system [17], and some dorsal root ganglion cells [18]. In addition, a recent study [14] has shown AQP1 is heavily expressed in a population of small diameter primary sensory neurons of dorsal root, trigeminal, and nodose ganglia. AQP1 immunoreactivity is abundant in cell bodies of the dorsal root ganglia and in both the peripheral and central branches of primary afferent neurons, and colocalized with markers of nociceptors, notably substance P, and IB4. Shields et al. [14] also found that a subset of neurons in the nodose ganglia indeed express AQP1 immunoreactivity, and that terminal staining is also observed in the nucleus of the solitary tract. By contrast, they found no neuronal AQP1 immunoreactivity in the superior cervical ganglion, which contains postganglionic sympathetic efferents, although capillary endothelium within this ganglion did immunostain positively for AQP1.

In a recent study, Oshio et al. [18] reported the presence of AQP1 immunoreactivity in superficial dorsal horn and primary afferent neurons of the dorsal root ganglia. They reported that AQP1 knockout mice had blunted nociceptive responses after intraplantar injection of capsaicin and longer response latencies in the tail flick assay of thermal nociception. They found strong AQP1 immunoreactivity in the superficial laminae I and II of the dorsal horn in the spinal cord of wild-type mice $(\mathrm{AQP} 1+/+)$. They suggested that this pattern of AQP1 expression is of interest because it is characteristic of nociceptive, small diameter primary afferent nerve fibers. Shields et al. [14] found that AQP1 was highly concentrated in unmyelinated axons, which was confirmed by the minimal overlap of AQP1 immunoreactivity with labeling for neurofilament 200, a marker of cell bodies with myelinated axons. They also demonstrated that AQP1 was localized to the plasma membrane of axons and synaptic terminals in the superficial dorsal horn, where it presumably can function as a native water channel. In the present study, we confirmed this immunolocalization of the AQP1 in the spinal cord. Shields et al. [14] also found that sciatic nerve injury altered AQP1 immunoreactivity in primary afferent terminals in the dorsal horn, neither electrophysiological nor a very complete behavioral analysis uncovered differences in nociceptive processing between mice lacking AQP1 and wild-type mice. Our results may be attributed AQP1 play a role in nociceptive processing after sciatic nerve injury.

Although functional studies using knockout mice have indicated the involvement of AQP1 in pain perception in the peripheral nervous system [12], some studies generated conflicting results where AQP1 is not required for normal pain processing $[10,14,19]$. Interestingly, the AQP1 null mutant mice appear to have normal nociceptive processing. The majority of fine cutaneous afferents in the mouse penetrate the superficial dorsal horn and make synaptic contacts at embryonic day 15.5 [20]. Shields et al. [14] concluded that despite the abundant and restricted expression of AQP1 in nociceptive primary afferent neurons, this channel is not required for normal pain processing. However, Wang et al. [21] found that AQP1 protein levels substantially increased after facial nerve injury. The change of AQP1 expression in the level of protein and mRNA highly coincided with the degree of the nerve edema in the facial canal.

Using optical absorbance method, Solenov et al. [22] reported swelling of the dorsal horn of spinal cord slices (300 $\mu \mathrm{m}$ thickness) in response to an osmotic challenge. Osmotic swelling in the dorsal horn, known to participate in nociceptive processing, was reduced in $\mathrm{AQP} 1-/-$ mice. In our study, induction of the cut injury resulted in 3.5-, 63-, and 4folds increase in the AQP1 intensity of fluorescence in the dorsal horn of the spinal cord at the 2nd, 24th, and 48th postoperative hours compared to intact group, respectively. Crush injury induced a slow increase in AQP1 immunofluorescence intensity in comparison to cut injury groups. A 3.4-, 30-, 2.5-folds increase in the AQP1 intensity of fluorescence were observed in the 2nd, 24th, and 48th postoperative hours compared to the intact group, respectively. Increase in the AQP1 immunoreactivity could be considered as a swelling after sciatic nerve injury as proposed by Solenov et al. [22] in mouse spinal cord. We also think that further studies should be carried to investigate mechanism of AQP1 in DRG and spinal cord in different injury types, postoperative periods, and experimental paradigms.

The aquaporins family in the central nervous system has diverse functions in neural signal transduction, cerebrospinal 
fluid formation, and osmoreception [4]. Maintenance of osmotic composition and volume within the interstitial, glial, and neuronal compartments are essential for normal function of the central nervous system [15]. Even small changes in osmolarity or volume can dramatically alter neuronal signaling and information processing [5,6]. Involvement of aquaporins in organellar physiology in vesicular swelling [23] and mitochondrial metabolism [24] has also been suggested. Sharp increase of the AQP1 immunoreactivity at the 24th postoperative hour and decrease at the 48 th postoperative hour may be attributed aforementioned neural signal transduction.

After sciatic nerve injury, it was found that molecular response on AQP1 immunoreactivity in dorsal root ganglia and their central axons connecting to the dorsal horn was significantly higher at the 24th postoperative hour. However, this intensive AQP1 response was not observed in the ventral horn. Intensive AQP1 expressions in synaptic buttons and astrocytic processes support the hypothesis according to which AQP1 expressions is involved in pain perception $[18,19,25]$. Involvement of the AQP1 in the regulation of synaptic vesicle swelling has also been reported [26]. After peripheral nerve trauma, increase in AQP1 immunoreactivity in the cytoplasm of the dorsal root ganglion cells may be related to speed of reorganization of the cytoplasma to the new event (trauma of the nerve). Peak in the AQP1 immunoreactivity at the 24th posttraumatic hour and then a rapid decrease in the 48th hour may be considered as tuning of the expression level of the AQP1 with pain inhibition or pain level. Increase in the AQP1 immunoreactivity may be related to increased synaptic activity leading to pain.

\section{Conclusion}

As a conclusion, we demonstrated that there was a temporal relationship with an increased expression of AQP1 following injury sustained to the sciatic nerve that was significantly observed in dorsal root ganglia and spinal cord. Those expressions were also subsided over time.

Acknowledgments This study was supported by Akdeniz University Research Fund (project number: 2013.01.0103.012). The authors also thank Mr. Erol NIZAMOGLU, Mr. Ibrahim CALISKAN, and Vet. Doga BESNE for their technical assistance.

\section{References}

1. Agre P (2004) Aquaporin water channels (Nobel Lecture). Angew Chem Int Ed Engl 43:4278-4290

2. King LS, Yasui M (2002) Aquaporins and disease: lessons from mice to humans. Trends Endocrinol Metab 13:355-360
3. Verkman AS (2000) Physiological importance of aquaporins: lessons from knockout mice. Curr Opin Nephrol Hypertens 9:517-522

4. Lehmann GL, Gradilone SA, Marinelli RA (2004) Aquaporin water channels in central nervous system. Curr Neurovasc Res 1:293-303

5. Castle NA (2005) Aquaporins as targets for drug discovery. Drug Discov Today 10:485-493

6. Huber VJ, Tsujita M, Nakada T (2012) Aquaporins in drug discovery and pharmacotherapy. Mol Asp Med 33:691-703

7. Badaut J, Hirt L, Granziera C, Bogousslavsky J, Magistretti PJ, Regli L (2001) Astrocyte-specific expression of aquaporin-9 in mouse brain is increased after transient focal cerebral ischemia. J Cereb Blood Flow Metab 21:477-482

8. Manley GT, Fujimura M, Ma T, Noshita N, Filiz F, Bollen AW, Chan P, Verkman AS (2000) Aquaporin-4 deletion in mice reduces brain edema after acute water intoxication and ischemic stroke. Nat Med 6: $159-163$

9. Nicchia GP, Frigeri A, Liuzzi GM, Svelto M (2003) Inhibition of aquaporin-4 expression in astrocytes by RNAi determines alteration in cell morphology, growth, and water transport and induces changes in ischemia-related genes. FASEB J 17:1508-1510

10. Oshio K, Watanabe H, Song Y, Verkman AS, Manley GT (2005) Reduced cerebrospinal fluid production and intracranial pressure in mice lacking choroid plexus water channel Aquaporin-1. FASEB J 19:76-78

11. Gao H, He C, Fang X, Hou X, Feng X, Yang H, Zhao X, Ma T (2006) Localization of aquaporin-1 water channel in glial cells of the human peripheral nervous system. Glia 53:783-787

12. Ma TH, Gao HW, Fang XD, Yang H (2011) Expression and function of aquaporins in peripheral nervous system. Acta Pharmacol Sin 32: 711-715

13. Nandasena BG, Suzuki A, Aita M, Kawano Y, Nozawa-Inoue K, Maeda T (2007) Immunolocalization of aquaporin-1 in the mechanoreceptive Ruffini endings in the periodontal ligament. Brain Res 1157:32-40

14. Shields SD, Mazario J, Skinner K, Basbaum AI (2007) Anatomical and functional analysis of aquaporin 1 , a water channel in primary afferent neurons. Pain 131:8-20

15. Rezzani R, Rodella LF (2010) Aquaporins and the nervous system: from bench to bedside. Curr Neuropharmacol 8:83

16. Kim IB, Lee EJ, Oh SJ, Park CB, Pow DV, Chun MH (2002) Light and electron microscopic analysis of aquaporin 1-likeimmunoreactive amacrine cells in the rat retina. J Comp Neurol 452:178-191

17. Nagahama M, Ma N, Semba R, Naruse S (2006) Aquaporin 1 immunoreactive enteric neurons in the rat ileum. Neurosci Lett 395: 206-210

18. Oshio K, Watanabe H, Yan D, Verkman AS, Manley GT (2006) Impaired pain sensation in mice lacking aquaporin-1 water channels. Biochem Biophys Res Commun 341:1022-1028

19. Zhang H, Verkman AS (2010) Aquaporin-1 tunes pain perception by interaction with $\mathrm{Na}(\mathrm{v}) 1.8 \mathrm{Na}+$ channels in dorsal root ganglion neurons. J Biol Chem 285:5896-5906

20. Ozaki S, Snider WD (1997) Initial trajectories of sensory axons toward laminar targets in the developing mouse spinal cord. J Comp Neurol 380:215-229

21. Wang H, Fang F, Yi J, Xiang Z, Sun M, Jiang H (2012) Establishment and assessment of the perinatal mouse facial nerve axotomy model via a subauricular incision approach. Exp Biol Med (Maywood) 237: 1249-1255

22. Solenov EI, Vetrivel L, Oshio K, Manley GT, Verkman AS (2002) Optical measurement of swelling and water transport in spinal cord slices from aquaporin null mice. J Neurosci Methods 113:85-90

23. Cho SJ, Sattar AK, Jeong EH, Satchi M, Cho JA, Dash S, Mayes MS, Stromer MH, Jena BP (2002) Aquaporin 1 regulates GTP-induced rapid gating of water in secretory vesicles. Proc Natl Acad Sci U S A 99:4720-4724 
24. Calamita G, Ferri D, Gena P, Liquori GE, Cavalier A, Thomas D, Svelto M (2005) The inner mitochondrial membrane has aquaporin-8 water channels and is highly permeable to water. $\mathrm{J}$ Biol Chem 280:17149-17153
25. Borsani E (2010) Aquaporins in sensory and pain transmission. Curr Neuropharmacol 8:122-127

26. Jeremic A, Cho WJ, Jena BP (2005) Involvement of water channels in synaptic vesicle swelling. Exp Biol Med (Maywood) 230:674-680 